\title{
Dynamic changes in fibrinogen and D-dimer levels in COVID-19 patients on nafamostat mesylate
}

\author{
Itsuki Osawa ${ }^{1} \cdot$ Koh Okamoto $^{1}$ (1) $\cdot$ Mahoko Ikeda $^{1,2} \cdot$ Amato Otani $^{1} \cdot$ Yuji Wakimoto ${ }^{1} \cdot$ Marie Yamashita $^{1}$. \\ Takayuki Shinohara $^{1} \cdot$ Yoshiaki Kanno $^{1}$ - Daisuke Jubishi ${ }^{1}$ - Makoto Kurano ${ }^{3}$. Sohei Harada ${ }^{1,2}$. Shu Okugawa ${ }^{1}$. \\ Yutaka Yatomi $^{3} \cdot$ Kyoji Moriya $^{1,2}$
}

Accepted: 3 September 2020 / Published online: 12 September 2020

(c) Springer Science+Business Media, LLC, part of Springer Nature 2020

\begin{abstract}
Critical illnesses associated with coronavirus disease 2019 (COVID-19) are attributable to a hypercoagulable status. There is limited knowledge regarding the dynamic changes in coagulation factors among COVID-19 patients on nafamostat mesylate, a potential therapeutic anticoagulant for COVID-19. First, we retrospectively conducted a cluster analysis based on clinical characteristics on admission to identify latent subgroups among fifteen patients with COVID-19 on nafamostat mesylate at the University of Tokyo Hospital, Japan, between April 6 and May 31, 2020. Next, we delineated the characteristics of all patients as well as COVID-19-patient subgroups and compared dynamic changes in coagulation factors among each subgroup. The subsequent dynamic changes in fibrinogen and D-dimer levels were presented graphically. All COVID-19 patients were classified into three subgroups: clusters A, B, and C, representing low, intermediate, and high risk of poor outcomes, respectively. All patients were alive 30 days from symptom onset. No patient in cluster A required mechanical ventilation; however, all patients in cluster $\mathrm{C}$ required mechanical ventilation, and half of them were treated with venovenous extracorporeal membrane oxygenation. All patients in cluster A maintained low D-dimer levels, but some critical patients in clusters B and C showed dynamic changes in fibrinogen and D-dimer levels. Although the potential of nafamostat mesylate needs to be evaluated in randomized clinical trials, admission characteristics of patients with COVID-19 could predict subsequent coagulopathy.
\end{abstract}

Keywords COVID-19 $\cdot$ Anticoagulant $\cdot$ D-dimer $\cdot$ Fibrinogen $\cdot$ Nafamostat mesylate

\section{Highlights}

- All patients with COVID-19 treated on nafamostat mesylate were alive at 30 days from symptom onset.

- Using cluster analysis based on clinical characteristics on admission, we identified three subgroups among COVID19 patients with different clinical presentations of subsequent coagulopathy.

\section{Koh Okamoto}

kokamoto-tky@umin.ac.jp

1 Department of Infectious Diseases, The University of Tokyo Hospital, 7-3-1 Hongo, Bunkyo-ku, Tokyo 113-0033, Japan

2 Department of Infection Control and Prevention, The University of Tokyo Hospital, Tokyo, Japan

3 Department of Clinical Laboratory, The University of Tokyo Hospital, Tokyo, Japan
- Clinical characteristics on admission are beneficial in predicting subsequent coagulopathy and consider individualized approaches for thromboembolism.

- Since COVID-19-associated coagulopathy resembles DIC, a careful evaluation of dynamic changes in coagulopathy, as reflected by fibrinogen and D-dimer levels, is essential.

\section{Introduction}

Critically ill patients with coronavirus disease 2019 (COVID19), caused by the severe acute respiratory syndrome coronavirus 2 (SARS-CoV-2), primarily present with respiratory failure [1]. However, another distinct feature of COVID-19 is its coagulopathy [2,3]. Coagulopathy, notably increased D-dimer levels, is commonly observed, even on admission, among patients with COVID-19 and is associated with subsequent thromboembolic events and severe outcomes [2-6]. 
Although the most effective strategy for treating coagulopathy is yet to be established, the International Society of Thrombosis and Haemostasis (ISTH) released an interim guidance that recommended consideration of anticoagulant therapy for all COVID-19 patients, based on limited evidence [7-9].

Although a variety of antiviral agents for COVID-19 has been studied extensively, there has been only one agent, remdesivir, that has proven to be superior to placebo in randomized controlled trials [10,11]. Among potential agents, nafamostat mesylate (hereafter nafamostat), a serine protease inhibitor, could be one of the promising agents for treating COVID-19. Nafamostat, which provides sufficient prolongation of activated partial thromboplastin time (APTT) without causing major bleeding complications, has been used as an anticoagulant for hemodialysis procedures and disseminated intravascular coagulation (DIC) in Japan and South Korea $[12,13]$. Previous in vitro studies revealed that nafamostat blocks the entry of SARS-CoV-2 into the human cell by preventing the fusion of the envelope of SARS-CoV-2 with the host cell $[14,15]$. Considering its antiviral and anticoagulant effects, nafamostat may effectively treat COVID-19 patients, particularly those who present with coagulopathy [16]. In light of this, we recently reported a case-series study where 11 critically ill patients with COVID-19 were successfully treated with nafamostat in combination with favipiravir, [17] another antiviral candidate that potentially exhibits activity against SARS-CoV-2 through inhibition of RNA-dependent RNA polymerase [18]. The efficacy of nafamostat alone or in combination with other agents is being evaluated in several phase three randomized trials (jRCTs031200026, NCT04418128, and NCT04352400).

Although many reports have focused on abnormal coagulation parameters on admission, a few others have reported on the subsequent changes in coagulation factors and their association with patient outcomes [4, 19-22]. In this study, we aimed to (1) use clustering analysis to identify the latent subgroups among COVID-19 patients based on their admission characteristics and (2) describe the differences in the subsequent changes in fibrinogen and D-dimer levels among the clusters, while on treatment with nafamostat and favipiravir, to inform strategies that could better control coagulopathy in COVID-19 patients.

\section{Methods}

\section{Study design and population}

This retrospective descriptive study was conducted at the University of Tokyo Hospital, a tertiary medical center in Tokyo, Japan. We included patients with COVID-19 (aged $\geq 18$ years) who were hospitalized between April
6 and May 31, 2020, and treated with nafamostat (a dose of $0.20 \mathrm{mg} / \mathrm{kg}$ ) and favipiravir (a dose of $3600 \mathrm{mg}$ on day 1 and, subsequently, $1600 \mathrm{mg}$ per day) for 14 days or until clinically improved. Additional methylprednisolone for acute respiratory distress syndrome as well as anticoagulants (i.e., intravenous heparin or direct oral anticoagulants) were used at the discretion of each physician. We excluded patients who were on comfort measures. All data were retrospectively retrieved from electronic health records. The presence of SARS-CoV-2 in sputum or nasopharyngeal-swab samples was confirmed using real-time polymerase chain reaction (RT-PCR; Roche, Switzerland). In addition, patients were tested again before discharge when their fever and respiratory symptoms had resolved, because the Japanese governmental policy required two consecutive negative RT-PCR assays before discharge from the hospital to confirm the viral clearance during the study period. The Institutional Review Board of the University of Tokyo approved this study (\#3538) and the Institutional Treatment Board of the University of Tokyo Hospital approved the treatment protocol for COVID-19 (\#202001CL). The informed consent was obtained from all patients.

\section{Statistical analysis}

\section{Cluster analysis}

To identify the latent subgroups among COVID-19 patients on admission, we used partitioning around medoids, which is a machine-learning-based clustering approach. On admission, the following variables were used for clustering: demographics [i.e., age, sex, body mass index (BMI), and days from symptom onset to admission], coexisting disorders (i.e., diabetes mellitus, hyperlipidemia, and hypertension), and laboratory data [i.e., $\mathrm{PaO}_{2} / \mathrm{FiO}_{2}$ ratio, white blood cell (WBC) count, the ratio of lymphocyte to total WBC count, hemoglobin $(\mathrm{Hb})$, platelet count, aspartate aminotransferase (AST), alanine aminotransferase (ALT), total bilirubin (T-Bil), lactate dehydrogenase (LDH), blood urea nitrogen (BUN), serum creatinine (SCr), C-reactive protein (CRP), prothrombin time (PT), APTT, fibrinogen, and D-dimer], which are well-known predictors of severe COVID-19 outcomes, according to past studies [22, 23]. We used Gower's distance measure to calculate distances between variables and determined the optimal number of clusters using the consensus matrix heatmaps as well as the consensus clustering methods (i.e., the elbow method and cumulative distribution function) [24]. In addition, we visually evaluated the clusters using the t-distributed stochastic neighbor embedding (t-SNE) method [25]. 


\section{Comparisons of COVID-19 subgroups treated with nafamostat}

We defined the outcomes of each subgroup as (1) mortality within 30 days from symptom onset, (2) days from admission to two consecutive negative RT-PCR results, and (3) days from symptom onset to two consecutive negative RT-PCR results. To delineate and compare the characteristics, treatments (i.e., the use of favipiravir, methylprednisolone, anticoagulants [i.e., heparin or direct oral

Table 1 Characteristics on admission, treatments, and outcomes of all patients treated with nafamostat mesylate and each subgroup

\begin{tabular}{|c|c|c|c|c|c|}
\hline & $\begin{array}{l}\text { Overall } \\
\mathrm{n}=15\end{array}$ & $\begin{array}{l}\text { Cluster A } \\
\mathrm{n}=4\end{array}$ & $\begin{array}{l}\text { Cluster B } \\
\mathrm{n}=5\end{array}$ & $\begin{array}{l}\text { Cluster C } \\
\mathrm{n}=6\end{array}$ & $\mathrm{p}$ value \\
\hline \multicolumn{6}{|l|}{ Demographics } \\
\hline Age, median (IQR) & $63.0(60.0-69.0)$ & $62.5(58.3-64.5)$ & $69.0(68.0-76.0)$ & $60.0(51.3-66.5)$ & 0.138 \\
\hline Male sex & $13(86.7)$ & $4(100.0)$ & $3(60.0)$ & $6(100.0)$ & 0.152 \\
\hline BMI, median (IQR) & $23.5(22.3-26.7)$ & $23.7(22.9-25.0)$ & $22.3(22.3-23.5)$ & $25.5(23.8-28.6)$ & 0.515 \\
\hline Days from symptom onset to admission (days) (IQR) & $8.00(7.00-11.0)$ & $8.00(7.25-9.00)$ & $7.00(7.00-11.0)$ & $9.50(8.25-10.8)$ & 0.798 \\
\hline \multicolumn{6}{|l|}{ Coexisting disorders } \\
\hline Diabetes mellitus & $6(40.0)$ & $0(0.0)$ & $5(100.0)$ & $1(16.7)$ & 0.002 \\
\hline Hyperlipidemia & $3(20.0)$ & $0(0.0)$ & $1(20.0)$ & $2(33.3)$ & 0.736 \\
\hline Hypertension & $6(40.0)$ & $0(0.0)$ & $2(40.0)$ & $4(66.7)$ & 0.141 \\
\hline \multicolumn{6}{|l|}{ Laboratory data } \\
\hline $\mathrm{PaO}_{2} / \mathrm{FiO}_{2}$, median (IQR) & $160(109-241)$ & $262(21-293)$ & $202(160-228)$ & $101(84.5-120)$ & 0.034 \\
\hline WBC count $\left(/ \mathrm{mm}^{3}\right)$, median $(\mathrm{IQR})$ & $6.00(5.05-8.30)$ & $4.90(4.15-6.22)$ & $5.60(4.70-6.60)$ & $9.05(6.55-11.3)$ & 0.095 \\
\hline The ratio of lymphocyte to total WBC (\%), median (IQR) & $14.7(11.0-18.9)$ & $17.6(14.6-20.7)$ & $14.7(13.2-17.6)$ & $11.1(8.93-16.6)$ & 0.433 \\
\hline Hemoglobin (g/dL), median (IQR) & $15.0(13.5-16.4)$ & $13.6(13.1-14.5)$ & $13.8(12.6-16.4)$ & $16.2(15.2-16.4)$ & 0.183 \\
\hline Platelet count $\left(\times 10^{4} / \mathrm{mm}^{3}\right)$, median $(\mathrm{IQR})$ & $20.0(18.0-27.9)$ & $20.4(17.2-23.3)$ & $19.6(16.5-25.1)$ & $25.3(19.4-30.6)$ & 0.386 \\
\hline AST (U/L), median (IQR) & $50.0(41.0-55.5)$ & $53.0(44.0-72.0)$ & $45.0(39.0-49.0)$ & $54.5(45.8-58.0)$ & 0.328 \\
\hline ALT (U/L), median (IQR) & $38.0(24.5-55.0)$ & $57.0(47.5-58.8)$ & $35.0(20.0-38.0)$ & $41.0(27.5-52.3)$ & 0.204 \\
\hline T-Bil (mg/dL), median (IQR) & $0.80(0.50-0.85)$ & $0.60(0.50-0.72)$ & $0.80(0.50-0.90)$ & $0.80(0.65-1.32)$ & 0.50 \\
\hline LDH (U/L), median (IQR) & $423(362-592)$ & $267(200-416)$ & $406(386-410)$ & $606(462-781)$ & 0.028 \\
\hline BUN (mg/dL), median (IQR) & $19.30(14.9-27.6)$ & $20.9(13.6-27.7)$ & $21.3(15.4-27.6)$ & $19.1(17.0-21.9)$ & 0.934 \\
\hline $\mathrm{SCr}(\mathrm{mg} / \mathrm{dL})$, median (IQR) & $0.92(0.71-1.03)$ & $0.97(0.91-1.06)$ & $0.92(0.74-0.92)$ & $0.76(0.59-1.01)$ & 0.543 \\
\hline CRP (mg/dL), median (IQR) & $10.5(5.42-13.4)$ & $4.36(1.43-8.06)$ & $6.08(4.76-12.2)$ & $16.2(15.2-16.4)$ & 0.033 \\
\hline PT (seconds), median (IQR) & $12.5(11.8-13.5)$ & $11.7(11.3-12.0)$ & $12.1(11.9-13.0)$ & $13.50(12.7-13.9)$ & 0.077 \\
\hline APTT (seconds), median (IQR) & $28.8(28.2-31.3)$ & $28.5(28.2-28.7)$ & $31.9(31.7-32.3)$ & $28.2(28.0-28.7)$ & 0.009 \\
\hline Fibrinogen (mg/dL), median (IQR) & $619(557-683)$ & $605(574-641)$ & $572(493-761)$ & $632(569-686)$ & 0.99 \\
\hline D-dimer $(\mu \mathrm{g} / \mathrm{mL})$, median (IQR) & $1.30(0.95-2.40)$ & $1.00(0.65-1.83)$ & $1.10(1.10-1.20)$ & $2.60(1.40-9.72)$ & 0.078 \\
\hline \multicolumn{6}{|l|}{ Treatments $^{\mathrm{a}}$} \\
\hline Favipiravir & $15(100.0)$ & $4(100.0)$ & $5(100.0)$ & $6(100.0)$ & 0.99 \\
\hline Methylprednisolone & $7(46.7)$ & $2(50.0)$ & $0(0.0)$ & $5(83.3)$ & 0.022 \\
\hline Anticoagulants (Heparin or DOAC) & $11(73.3)$ & $1(25.0)$ & $3(60.0)$ & $6(100.0)$ & 0.165 \\
\hline Mechanical ventilation & $7(46.7)$ & $0(0.0)$ & $1(20.0)$ & $6(100.0)$ & 0.001 \\
\hline VV-ECMO & $3(20.0)$ & $0(0.0)$ & $0(0.0)$ & $3(50.0)$ & 0.075 \\
\hline \multicolumn{6}{|l|}{ Outcomes $^{\mathrm{a}}$} \\
\hline Mortality within 30 days from symptom onset & $0(0.0)$ & $0(0.0)$ & $0(0.0)$ & $0(0.0)$ & 0.99 \\
\hline $\begin{array}{l}\text { Days from admission to } 2 \text { consecutive negative PCR (days), } \\
\text { median (IQR) }\end{array}$ & $14.5(10.0-20.8)$ & $17.0(13.0-21.3)$ & $12.5(8.75-17.3)$ & $13.0(10.3-19.5)$ & 0.620 \\
\hline $\begin{array}{l}\text { Days from symptom onset to } 2 \text { consecutive negative PCR (days), } \\
\text { median (IQR) }\end{array}$ & $23.0(21.0-27.8)$ & $23.5(22.0-27.0)$ & $23.5(18.3-28.8)$ & $22.5(21.0-26.3)$ & 0.684 \\
\hline
\end{tabular}

$B M I$ body mass index, $W B C$ white blood cells, $A S T$ aspartate aminotransferase, $A L T$ alanine aminotransferase, $T$-Bil total bilirubin, $L D H$ lactate dehydrogenase, $B U N$ blood urea nitrogen, $S C r$ serum creatinine, $C R P$ C-reactive protein, $P T$ prothrombin time, $A P T T$ activated partial thrombin time, $D O A C$ direct oral anticoagulants, $V V$-ECMO venovenous extracorporeal membrane oxygenation, $P C R$ polymerase chain reaction, $I Q R$ interquartile range

${ }^{a}$ Variables not used for cluster analysis

${ }^{\mathrm{b}}$ One patient in cluster $\mathrm{C}$ was excluded only from these analyses since the PCR result did not turn negative by 31 th May, 2020 (35 days after onset and 30 days after admission)

Values represent $\mathrm{n}(\%)$, unless otherwise indicated 
anticoagulants], mechanical ventilation, and venovenous extracorporeal membrane oxygenation [VV-ECMO]), and outcomes of each cluster, we used Kruskal-Wallis and Fisher's tests, as appropriate. We constructed line graphs to visualize dynamic changes in fibrinogen and D-dimer levels in patients with COVID-19 and associated coagulopathy from each cluster. In addition, we graphically displayed sequential changes in D-dimer/fibrinogen ratio, a specific marker for embolism [26, 27].

The results of two-tailed tests with $\mathrm{P}<.05$ were considered to be statistically significant. All analyses were conducted using R version 3.6.1 (R Foundation, Vienna, Austria).

\section{Results}

Among the 26 patients who were hospitalized, 16 were treated with nafamostat and favipiravir, 6 with favipiravir (primarily due to the limited supply of nafamostat), and 4 without any antiviral agents. One patient on nafamostat and favipiravir and another on no antiviral agents were transitioned to comfort measures soon after admission; thus, they were excluded from the analysis. Among 15 patients in the final cohort (Table 1), the median age was 63.0 years and 13 patients were men. Twelve, seven, and three patients out of fifteen required intensive care, mechanical ventilation, and VV-ECMO, respectively. No patients died nor developed thromboembolic events 30 days from symptom onset. Three patients developed phlebitis at the peripheral intravenous site secondary to nafamostat treatment.

In terms of the clustering approach, we found that the three-class model was optimal, judging from the consensus matrix heatmaps, elbow method, cumulative distribution function curve, and t-SNE plot (Figs. 1, 2). The characteristics and outcomes of all patients and clusters are presented in Table 1. In summary, the patients who belonged to cluster A had high $\mathrm{PaO}_{2} / \mathrm{FiO}_{2}$ ratios and low $\mathrm{LDH}, \mathrm{CRP}$, and D-dimer levels. The patients who belonged to cluster $\mathrm{C}$ were likely to have hyperlipidemia and hypertension. Further, they had low $\mathrm{PaO}_{2} / \mathrm{FiO}_{2}$ ratios; high WBC counts; and high $\mathrm{LDH}$, $\mathrm{CRP}$, and D-dimer levels. The levels of patients in cluster B measured between those of the patients in cluster A and cluster C. Accordingly, the patients in cluster A did not require mechanical ventilation and VV-ECMO; however, all patients in cluster $\mathrm{C}$ required mechanical ventilation and half of them were treated with VV-ECMO. Nevertheless, there was no significant difference in days from symptom onset or admission to two consecutive negative RT-PCR results among the three subgroups.

Clinical courses of coagulopathy, as reflected by changes in fibrinogen and D-dimer levels, in patients with COVID-19 from each cluster are shown in Fig. 3. In cluster A, D-dimer maintained a low level; however, some critically ill patients in clusters $\mathrm{B}$ and $\mathrm{C}$ showed dynamic changes in coagulation parameters (e.g., D-dimer level was increasing, while fibrinogen level was decreasing).

\section{Discussion}

Using cluster analysis, we statistically classified 15 patients with COVID-19 treated with nafamostat mesylate into 3 subgroups: cluster $\mathrm{A}, \mathrm{B}$, and $\mathrm{C}$. Given that low $\mathrm{PaO}_{2} / \mathrm{FiO}_{2}$ ratios as well as elevated LDH and D-dimer levels on admission are risk factors for poor outcomes in COVID-19 as per previous studies, [22,23] the risk for poor outcomes was expected to be low, intermediate, and high for clusters A, B, and C, respectively. In addition, some critical patients in clusters $\mathrm{B}$ and $\mathrm{C}$ (e.g., patient $\mathrm{C}-2$ on mechanical ventilation and VVECMO in Fig. 3) showed dynamic changes in coagulation parameters (i.e., fibrinogen and D-dimer levels).

Several studies to date have described the dynamic changes in coagulation factors during the course of illness [4, 19-22]. Although they summarized their original data in various ways, they consistently revealed a significantly dynamic difference in coagulation factors between survivors and non-survivors as well as a steady increase in D-dimer levels in the early phase (the first 2 weeks of the disease). We described the different patterns of dynamic changes
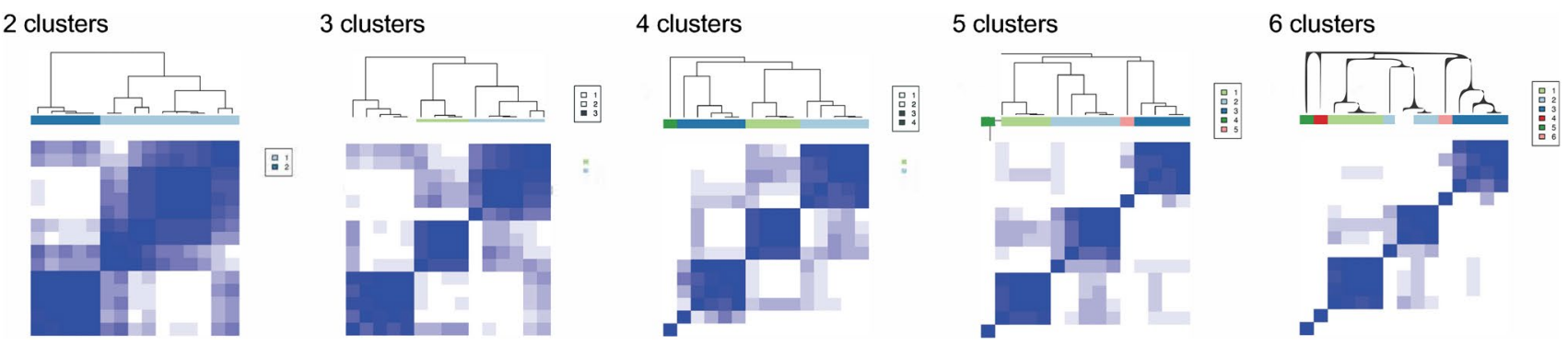

Fig. 1 Consensus matrix heatmaps for different numbers of clusters. The heatmaps, based on hierarchical clustering, indicate that patients treated with nafamostat mesylate could be categorized into $\geq$ three clusters with relatively clear boundaries 

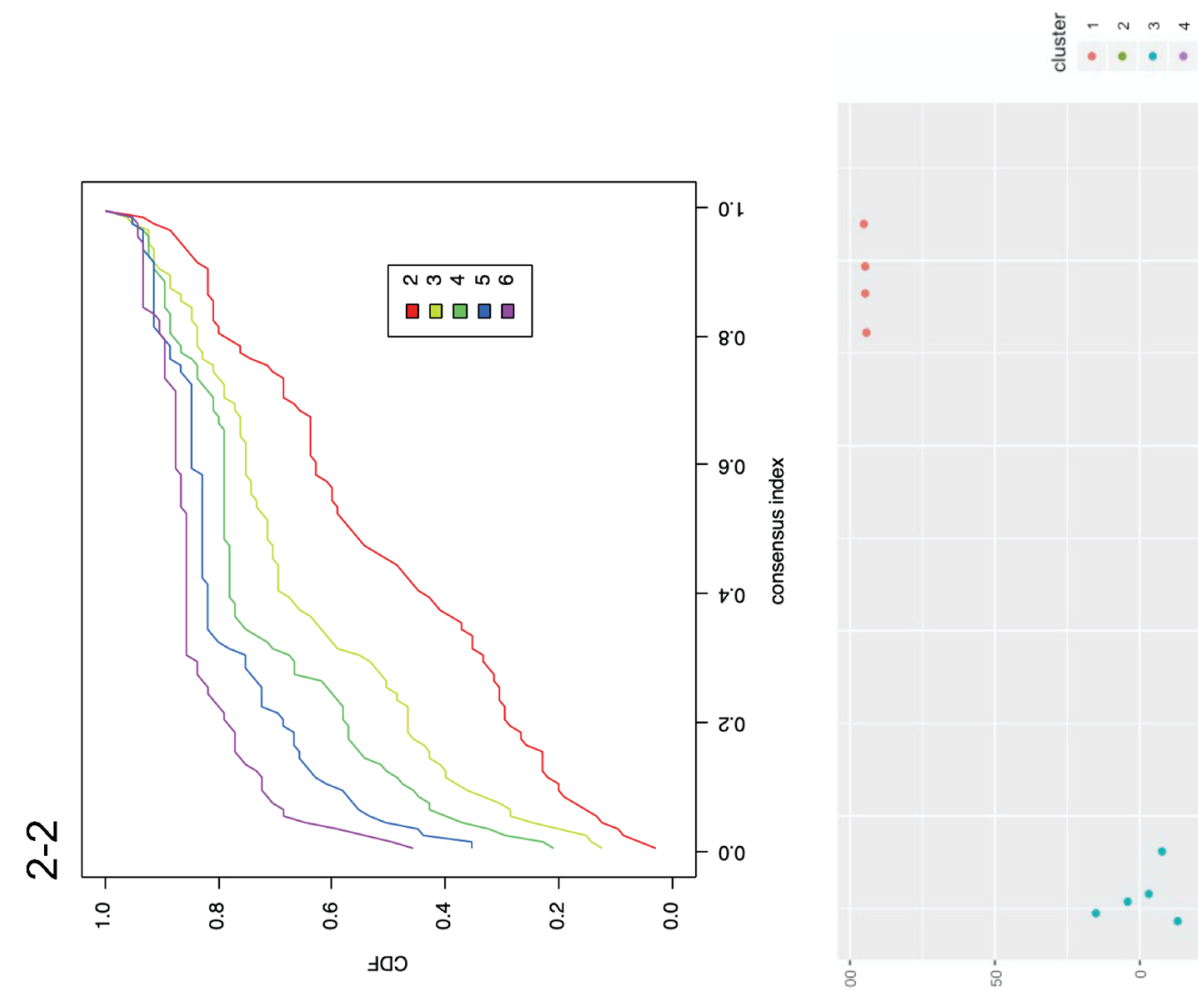

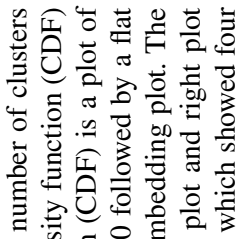

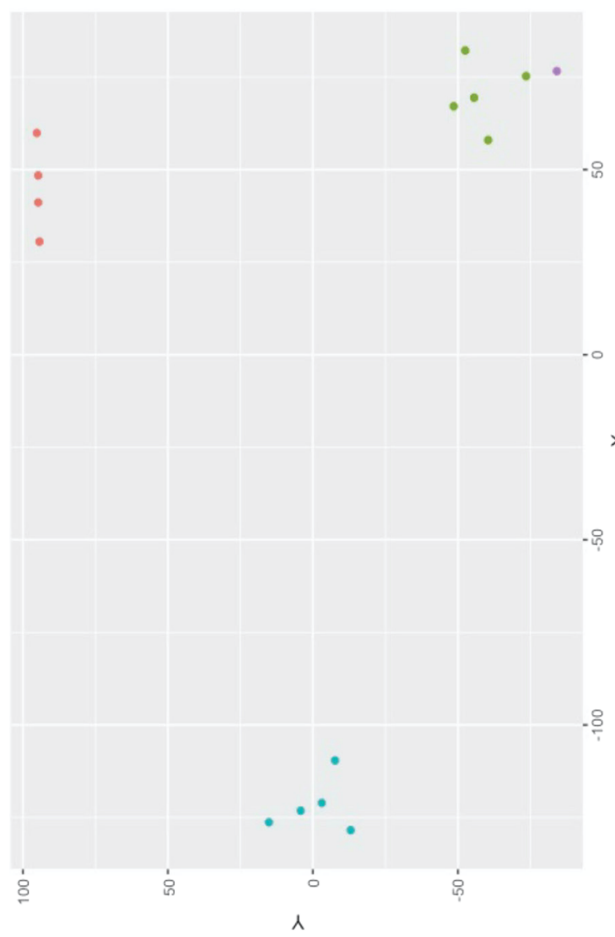

幽

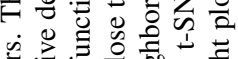

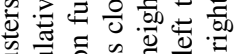

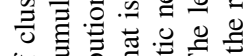

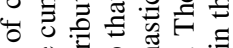

ठี

है 0 क क क

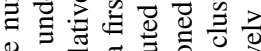

巳

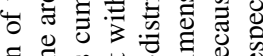

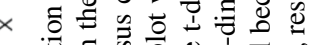

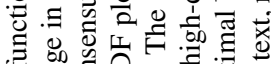

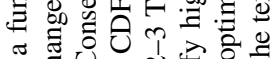

๘

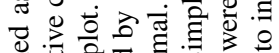

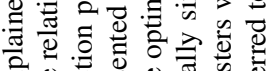

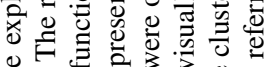

Uूं

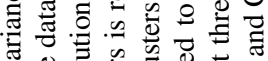

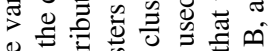

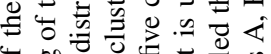

पे

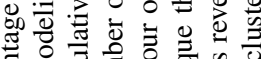

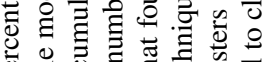

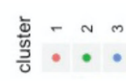

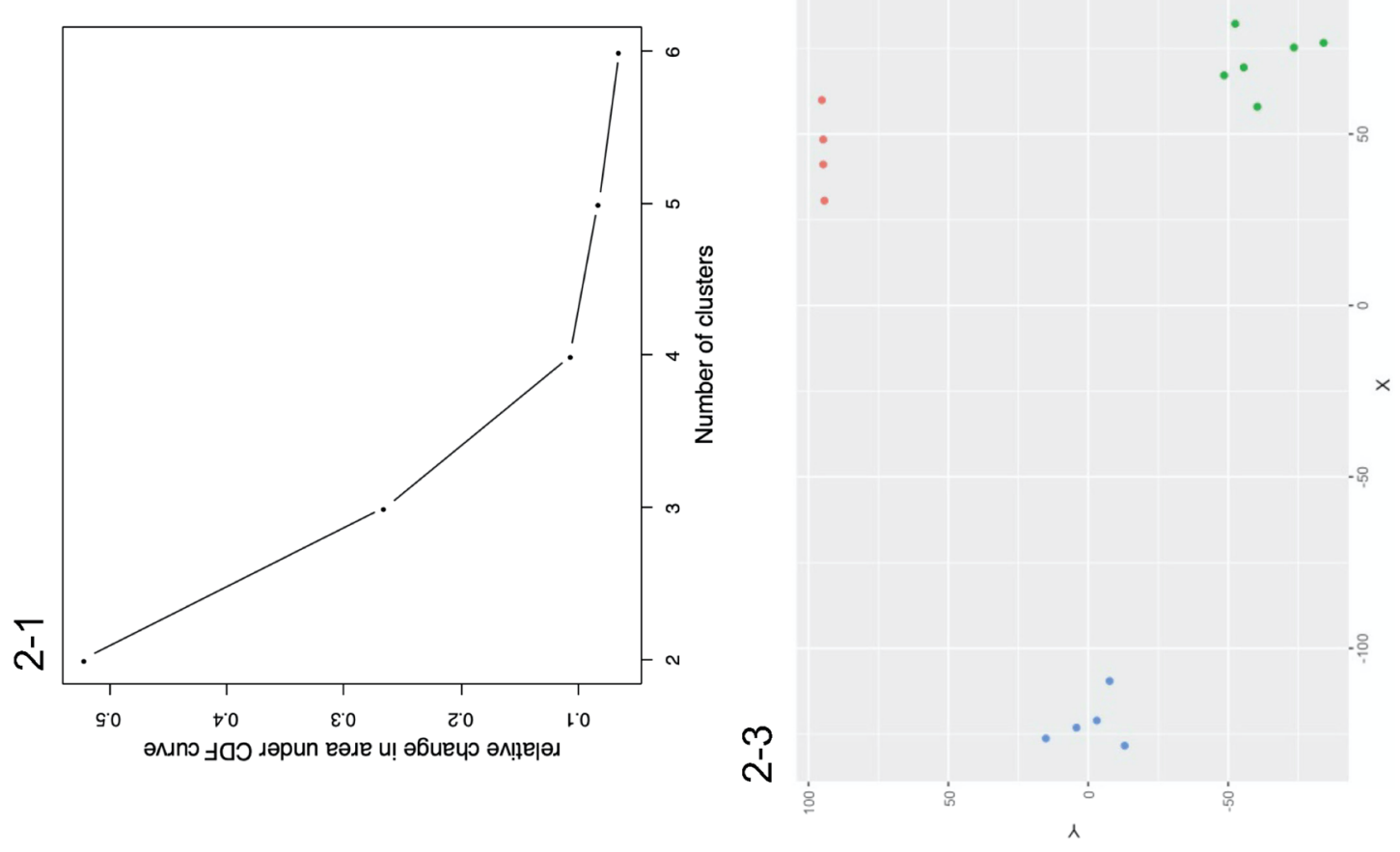

至

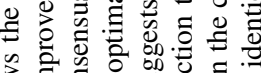

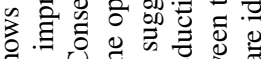
क त छ

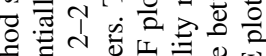

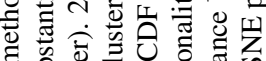

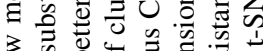

के के

\%

\& \&

ن

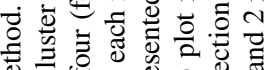

킁 유

घ ᄒᄒ

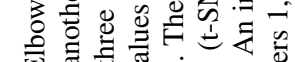

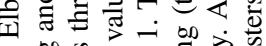

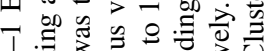

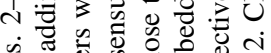

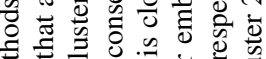

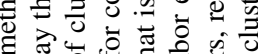

का $30=$ ची

毒

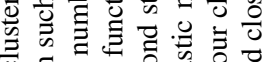

․ $\equiv$ o

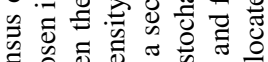

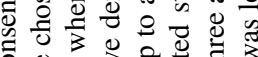

ర。

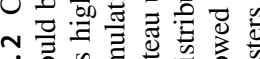

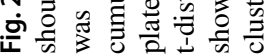




\section{min}

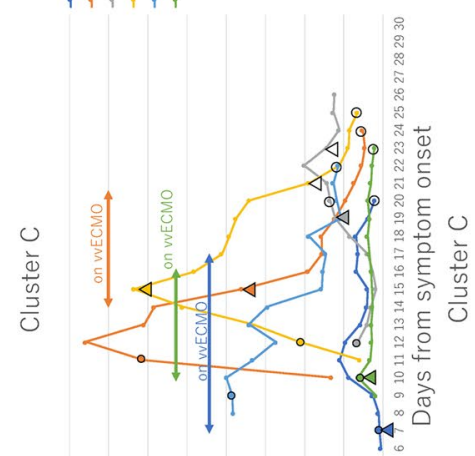

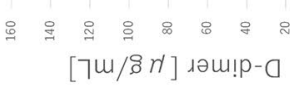

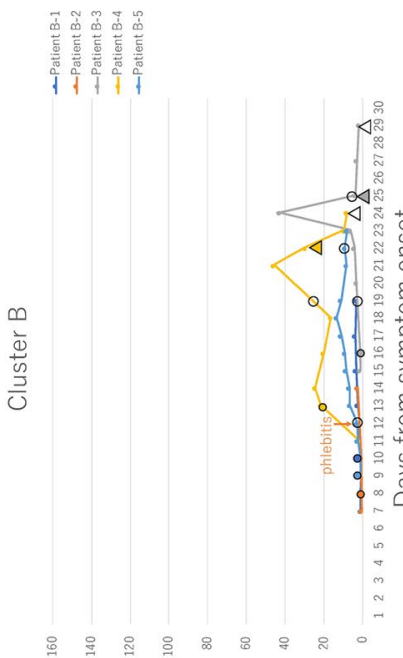

[7m/\&r]

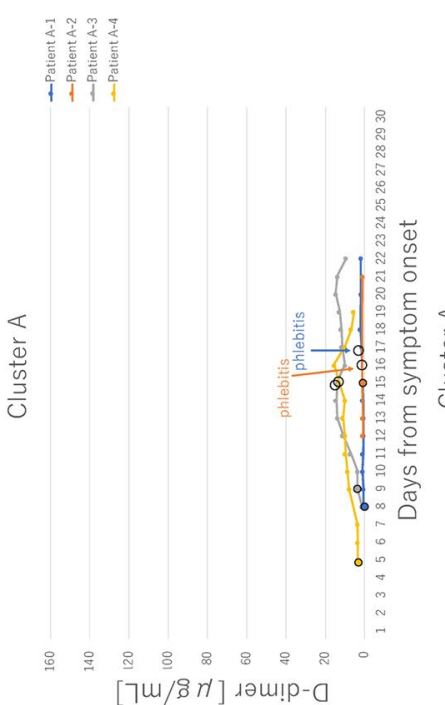

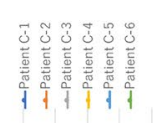

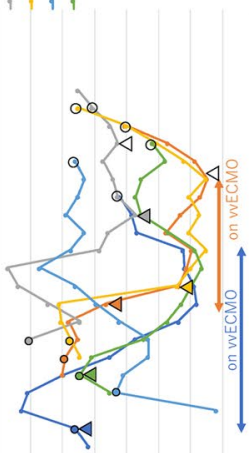

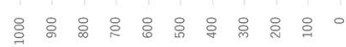

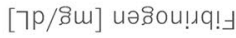

mint

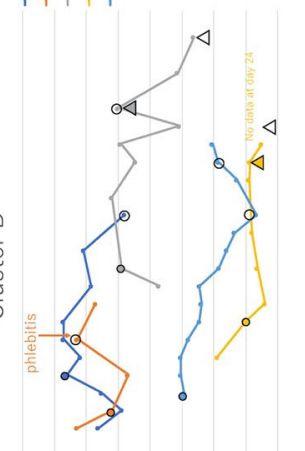

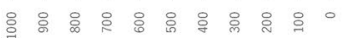

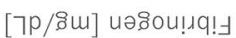<smiles>C1=C[As]=C1</smiles>

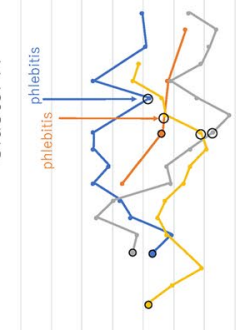

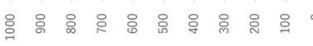

[าp/вแ] иәвоน!ıq!-

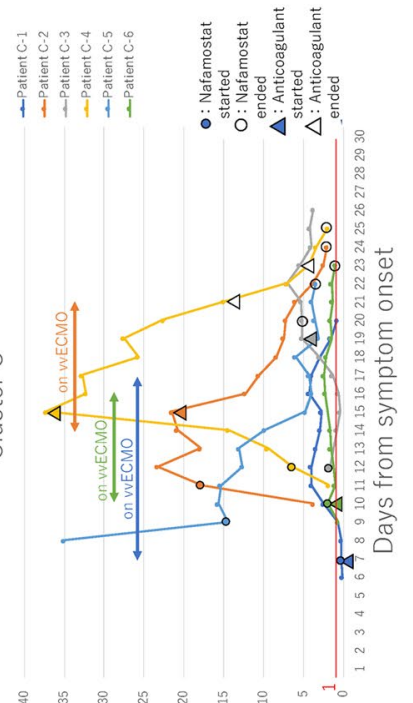

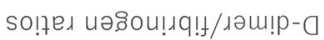

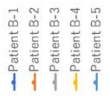

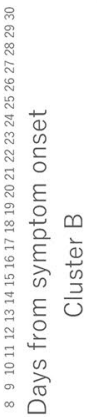

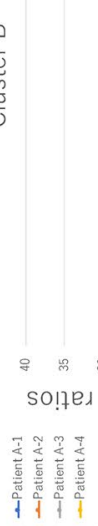

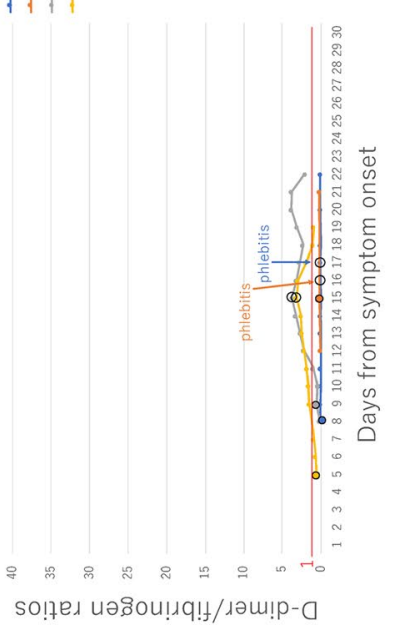

.

西

si

过

$\overrightarrow{0}$

可 官豆 $\Xi$ 过 os 踏 ठ 을 言官 ล․․

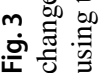


in coagulation parameters among three subgroups, which were statistically classified, based only on patient characteristics on admission and not on their outcomes. Given that some patients in clusters $\mathrm{B}$ and $\mathrm{C}$ had noticeable increases in D-dimer levels, patients with specific features (e.g., low $\mathrm{PaO}_{2} / \mathrm{FiO}_{2}$ ratios; high CRP; and history of diabetes mellitus, hypertension, and hyperlipidemia), even without elevated D-dimer levels on admission, may benefit from preemptive anticoagulation therapy to prevent progressive coagulopathy.

COVID-19-associated coagulopathy resembles DIC and is associated with enhanced fibrinolysis rather than suppressed fibrinolysis as with other infectious-disease-related DIC $[2,3,16]$. As Tang et al. observed that fibrinogen initially increased and then rapidly decreased about 10 days after admission in COVID-19 non-survivors, [4] there may be a transition point from suppressed to enhanced fibrinolysis in the clinical course of COVID-19. Our discovery of some critically ill patients in clusters B and C having an initial increase in D-dimer levels with a subsequent decrease in fibrinogen levels was consistent with Tang et al.'s observation, [4] and these dynamic changes in fibrinogen and D-dimer levels may indicate the development of thromboembolism and subsequent increased risk of hemorrhage. Wichmann et al. reported autopsies revealing that $58 \%$ of patients had deep venous thrombosis, unsuspected before death, [28] meaning that new thromboembolism could not be recognized, if not critical. A D-dimer/fibrinogen ratio, calculated using the formula: (D-dimer $[\mu \mathrm{g} / \mathrm{ml}] /$ fibrinogen $[\mathrm{mg} / \mathrm{dL}]) \times 100$, has proven to be a specific marker for embolism, [26, 27] and the ratio above 1.0 had $94 \%$ specificity in the diagnosis of pulmonary embolism in non-COVID-19 patients [26]. Indeed, as shown in Fig. 3, a high D-dimer/ fibrinogen ratio (above 1.0 in some patients) may also suggest clinically silent thromboembolism. Fortunately, no patient experienced any critical thromboembolic or hemorrhagic events as within 30 days from their symptom onset. Notwithstanding, monitoring the trend of this ratio as well as fibrinogen and D-dimer levels may be useful in the prediction and management of coagulopathy in COVID-19.

However, our study has the following limitations. First, it is a retrospective, single-arm descriptive study of fifteen patients with COVID-19 treated with nafamostat and favipiravir in an academic hospital. Therefore, it is uncertain if our favorable outcomes are primarily attributable to nafamostat and favipiravir. However, considering that the anti-SARS-CoV-2 effect of nafamostat has been suggested in vitro, $[14,15]$ its antiviral and anticoagulant activities could work synergistically against COVID-19 with coagulopathy $[16,17]$. Second, additional anticoagulants (i.e., intravenous heparin or direct oral anticoagulants), used on some patients, might have contributed to the dynamic changes in fibrinogen and D-dimer levels observed in this study. Third, while there is no definite rule for the minimum sample size for clustering approaches [29] and the indicative variables appear to be distributed appropriately, our small sample size requires extra caution when interpreting our findings. Further studies are needed to confirm our findings.

\section{Conclusions}

Using cluster analysis based on their admission status, we statistically classified patients with COVID-19 into three clusters that subsequently had different dynamic changes in fibrinogen and D-dimer levels. Individualized approachesthe use of prophylactic and enhanced anticoagulants when there is high risk of thromboembolism-are necessary together with the careful evaluation of dynamic changes in coagulopathy, as reflected by fibrinogen and D-dimer levels. Further research regarding the effectiveness of nafamostat against COVID-19 and its effects on COVID-19-associated coagulopathy is urgently necessary for guiding physicians towards the optimal treatment strategy.

Acknowledgements We appreciate all medical staff for their dedication and professionalism in the fight against COVID-19.

Author contributions IO and $\mathrm{KO}$ conceived and designed the study. All authors interpreted the data, critically revised the manuscript, and approved the final manuscript. IO performed statistical analyses, IO and $\mathrm{KO}$ drafted the initial manuscript, and KM supervised the study.

Funding We received no financial support from any organization for the submitted work.

\section{Compliance with ethical standards}

Conflict of interest Dr. Harada reports personal fees from BD, personal fees from Meiji, personal fees from Shionogi, personal fees from Sumitomo Dainippon Pharma, grants and personal fees from MSD, personal fees from Astellas, personal fees from Beckman Coulter Diagnostics, personal fees from FUJIFILM Toyama Chemical, outside the submitted work.

Ethical approval The Institutional Review Board of the University of Tokyo approved this study (\#3538) and the Institutional Treatment Board of the University of Tokyo Hospital approved the treatment protocol for COVID-19 (\#202001CL). Informed consent was obtained from all patients.

\section{References}

1. Huang C, Wang Y, Li X et al (2020) Clinical features of patients infected with 2019 novel coronavirus in Wuhan, China. The Lancet 395:497-506

2. Levi M, Thachil J, Iba T et al (2020) Coagulation abnormalities and thrombosis in patients with COVID-19. Lancet Haematol 7:e438-e440 
3. The Lancet $\mathrm{H}$ (2020) COVID-19 coagulopathy: an evolving story. Lancet Haematol. https://doi.org/10.1016/s2352-3026(20)30151 $-4$

4. Tang N, Li D, Wang X et al (2020) Abnormal coagulation parameters are associated with poor prognosis in patients with novel coronavirus pneumonia. J Thromb Haemost 18:844-847

5. Zhang L, Yan X, Fan Q et al (2020) D-dimer levels on admission to predict in-hospital mortality in patients with Covid-19. J Thromb Haemost. https://doi.org/10.1111/jth.14859

6. Wright FL, Vogler TO, Moore EE et al (2020) Fibrinolysis shutdown correlates to thromboembolic events in severe COVID-19 infection. J Am Coll Surg. https://doi.org/10.1016/j.jamcollsur g.2020.05.007

7. Thachil J, Tang N, Gando S et al (2020) ISTH interim guidance on recognition and management of coagulopathy in COVID-19. J Thromb Haemost 18:1023-1026

8. Connors JM, Levy JH (2020) Thromboinflammation and the hypercoagulability of COVID-19. J Thromb Haemost. https:// doi.org/10.1111/jth.14849

9. Tang N, Bai H, Chen X et al (2020) Anticoagulant treatment is associated with decreased mortality in severe coronavirus disease 2019 patients with coagulopathy. J Thromb Haemost 18:1094-1099

10. Beigel JH, Tomashek KM, Dodd LE et al (2020) Remdesivir for the treatment of Covid-19-preliminary report. N Engl J Med. https ://doi.org/10.1056/NEJMoa2007764

11. Wang Y, Zhang D, Du G et al (2020) Remdesivir in adults with severe COVID-19: a randomised, double-blind, placebo-controlled, multicentre trial. The Lancet 395:1569-1578

12. Choi JY, Kang YJ, Jang HM et al (2015) Nafamostat mesilate as an anticoagulant during continuous renal replacement therapy in patients with high bleeding risk: a randomized clinical trial. Medicine 94:e2392

13. Kimura F, Takahashi A, Kitazawa J et al (2020) Successful conservative treatment for massive uterine bleeding with non-septic disseminated intravascular coagulation after termination of early pregnancy in a woman with huge adenomyosis: case report. BMC Womens Health 20:56

14. Hoffmann M, Schroeder S, Kleine-Weber H et al (2020) Nafamostat mesylate blocks activation of SARS-CoV-2: new treatment option for COVID-19. Antimicrob Agents Chemother 64:e00754-e00720

15. Yamamoto M, Kiso M, Sakai-Tagawa Y et al (2020) The anticoagulant nafamostat potently inhibits SARS-CoV-2 S proteinmediated fusion in a cell fusion assay system and viral infection in vitro in a cell-type-dependent manner. Viruses 12:E629

16. Asakura H, Ogawa H (2020) Potential of heparin and nafamostat combination therapy for COVID-19. J Thromb Haemost 18:1521-1522
17. Doi K, Ikeda M, Hayase $\mathrm{N}$ et al (2020) Nafamostat mesylate treatment in combination with favipiravir for patients critically ill with Covid-19: a case series. Crit Care. https://doi.org/10.1186/s1305 4-020-03078-Z

18. Cai Q, Yang M, Liu D et al (2020) Experimental treatment with favipiravir for COVID-19: an open-label control study. Engineering. https://doi.org/10.1016/j.eng.2020.03.007

19. Wang D, Hu B, Hu C et al (2020) Clinical characteristics of 138 hospitalized patients with 2019 novel coronavirus-infected Pneumonia in Wuhan, China. JAMA. https://doi.org/10.1001/ jama.2020.1585

20. Li Y, Zhao K, Wei H et al (2020) Dynamic relationship between D-dimer and COVID-19 severity. Br J Haematol. https://doi. org/10.1111/bjh.16811

21. Fu J, Kong J, Wang W et al (2020) The clinical implication of dynamic neutrophil to lymphocyte ratio and D-dimer in COVID19: a retrospective study in Suzhou China. Thromb Res 192:3-8

22. Zhou F, Yu T, Du R et al (2020) Clinical course and risk factors for mortality of adult inpatients with COVID-19 in Wuhan, China: a retrospective cohort study. The Lancet 395:1054-1062

23. Wu C, Chen X, Cai Y et al (2020) Risk factors associated with acute respiratory distress syndrome and death in patients with coronavirus disease 2019 pneumonia in Wuhan, China. JAMA Intern Med. https://doi.org/10.1001/jamainternmed.2020.0994

24. Wilkerson MD, Hayes DN (2010) ConsensusClusterPlus: a class discovery tool with confidence assessments and item tracking. Bioinformatics 26:1572-1573

25. Maaten LVD, Hinton G (2008) Visualizing data using t-SNE. J Mach Learn Res 9:2579-605

26. Kara H, Bayir A, Degirmenci S et al (2014) D-dimer and D-dimer/ fibrinogen ratio in predicting pulmonary embolism in patients evaluated in a hospital emergency department. Acta Clin Belg 69:240-245

27. Hajsadeghi S, Kerman SR, Khojandi M et al (2012) Accuracy of D-dimer:fibrinogen ratio to diagnose pulmonary thromboembolism in patients admitted to intensive care units. Cardiovasc J Afr 23:446-456

28. Wichmann D, Sperhake JP, Lutgehetmann M et al (2020) Autopsy findings and venous thromboembolism in patients with COVID19. Ann Intern Med. https://doi.org/10.7326/M20-2003

29. Vidman L, Kallberg D, Ryden P (2019) Cluster analysis on high dimensional RNA-seq data with applications to cancer researchan evaluation study. PLoS ONE 14:e0219102

Publisher's Note Springer Nature remains neutral with regard to jurisdictional claims in published maps and institutional affiliations. 